Cahiers $d u$ MONDE RUSSE

\section{Cahiers du monde russe}

Russie - Empire russe - Union soviétique et États indépendants

$58 / 4 \mid 2017$

Varia

\title{
Fabien Bellat, Amériques URSS : Architectures du défi
}

\section{Vincent Hecquet}

\section{OpenEdition}

\section{Journals}

Édition électronique

URL : http://journals.openedition.org/monderusse/10183

DOI : $10.4000 /$ monderusse. 10183

ISSN : $1777-5388$

\section{Éditeur}

Éditions de l'EHESS

\section{Édition imprimée}

Date de publication : 1 octobre 2017

Pagination : 737-741

ISBN : 978-2-7132-2698-4

ISSN : $1252-6576$

Référence électronique

Vincent Hecquet, «Fabien Bellat, Amériques URSS : Architectures du défi », Cahiers du monde russe [En ligne], 58/4 | 2017, mis en ligne le 01 octobre 2017, consulté le 06 janvier 2021. URL : http:// journals.openedition.org/monderusse/10183; DOI : https://doi.org/10.4000/monderusse.10183 
Fabien BELLAT

\section{Amériques URSS}

Architectures du défi

Paris : Éditions Nicolas Chaudun, 2014, 304 p.

Spécialiste de l'architecture soviétique, Fabien Bellat étudie la façon dont l'URSS chercha à rivaliser avec les États-Unis dans le domaine architectural, par la 
construction d'immeubles prestigieux et notamment de gratte-ciel. Il étudie également les bâtiments que le régime soviétique fit édifier à l'étranger notamment sur le continent américain, ambassades et pavillons pour les expositions universelles. C'est donc à dessein que l'ouvrage n'aborde pas les réalisations de style constructiviste des années 1920 et 1930. De même, parmi les réalisations postérieures aux années 1950, l'auteur se concentre sur celles construites à l'étranger, après avoir consacré un remarquable article à l'architecture de la période brejnévienne ${ }^{1}$.

Dans les années 1920 et 1930, les réalisations inspirées par les immeubles américains furent peu nombreuses. Outre la pénurie de matériaux, les architectes soviétiques devaient composer avec l'idéologie officielle condamnant la ville capitaliste. Toutefois, ceci n'empêcha pas les contacts entre architectes soviétiques et occidentaux. L'Allemand Erich Mendelson (1887-1953) obtint en 1926 la commande de l'usine Drapeau rouge de Leningrad. Le Corbusier réalisa en 1930 le Centrosoyouz, Bureau central des coopératives, désormais siège de l'Institut statistique de Russie. En 1929, alors que les États-Unis n'avaient pas encore reconnu l'URSS, des architectes soviétiques purent concourir au projet de phare à la mémoire de Christophe Colomb à Saint-Domingue lancé par la Pan American Union. Symétriquement, des architectes étrangers participèrent en 1931 au concours pour le Palais des Soviets. En 1937, l'américain Frank Lloyd Wright (1867-1959) fut l'invité d'honneur du premier congrès des architectes soviétiques.

Cette ambivalence entre curiosité pour les réalisations étrangères et défiance idéologique trouve une tragique illustration dans le destin de Vjačeslav Oltarževskij (1880-1966). Envoyé en 1924 aux États-Unis pour une mission d'études officielle, il y demeura dix ans durant lesquels il collabora avec l'architecte américain Harvey Wiley Corbett (1873-1954) à l'époque où furent construits la plupart des gratte-ciel new-yorkais. Rentré volontairement en URSS, son plan directeur pour la construction du VDNH (Vystavka Dostiženij Narodnogo Hozjaistva - Exposition des réalisations de l'économie nationale) fut adopté. Toutefois, en 1938, dans la macabre mécanique de la terreur stalinienne, la chute de son commanditaire, le commissaire à l'Agriculture Mihail Černov, entraîna son arrestation. Černov fut exécuté et Oltarževskij envoyé dans un camp, où il reçut la faveur ambigüe de servir d'architecte en chef de la ville de Vorkuta. Ses bâtiments déjà réalisés au VDNH furent hâtivement démolis et remplacés par d'autres plus conformes au néoacadémisme désormais en vigueur. Libéré en 1943, Oltarževski fut cantonné à un rôle de spécialiste technique pour la construction des gratte-ciel staliniens. Il travailla notamment sous la direction d'Arkadij Mordvinov (1896-1964), promu lui au contraire à l'occasion des purges, avec qui il reçut l'honneur équivoque de partager en 1948 le prix Stalin pour la conception de l'hôtel Ukrajna.

Dans les années 1920 et 1930, l'influence des gratte-ciel américains sur les constructions soviétiques se borna à des «porosités et résonances formelles » (p. 15). Une des réalisations les plus réussies fut le bâtiment de Boris Iofan (1891-1976) pour l'exposition universelle de Paris en 1937 (remonté ensuite près du VDNH). Surmonté du groupe de Vera Muhina (1998-1953), L'ouvrier et la kolkhozienne, le bâtiment applique la formule des gratte-ciel de Chicago. Pour 
l'exposition internationale suivante, en 1939 à New York, Iofan dut partager la commande avec une étoile montante du stalinisme, Karo Alabjan (1897-1959). Leur collaboration déboucha sur une construction hybride, avec une tour à la manière d'Iofan qu'Alabjan enveloppa d'une structure semi-circulaire directement inspirée du pavillon Ford de l'exposition de Chicago en 1933-1934. Les halls des gratte-ciel américains influencèrent également l'intérieur de plusieurs stations du métro de Moscou. Aleksej Duškin (1904-1977) réalisa en 1935 la station Kropotkina dont la voûte dépouillée déguisant l'éclairage artificiel est inspirée par les galeries des temples égyptiens et la voûte du Louvre, mais aussi celle du hall du Singer Building construit en 1908 par Ernest Flagg (1857-1947) à New York (et détruit en 1968). Duškin réalisa aussi la station Majakovskaja ainsi que celle Plošad' Revoljucii dont les arcades sont garnies des célèbres statues en bronze de sportifs, de militaires et de femmes soviétiques de Matvej Manizer (1891-1966). Par la suite, Duškin se souvint encore des halls de gratte-ciel lorsqu'il conçut l'intérieur du ministère du Rail (1951) et le magasin de jouets Detskij Mir (ouvert en 1957) place de la Lubjanka.

Avec les débuts de la Guerre froide, le souci de rivaliser avec les États-Unis conduisit Stalin à décider la construction d'immeubles de grande hauteur à Moscou. Le conseil des ministres de l'URSS décréta le 13 janvier 1947 la construction de huit édifices « à étages multiples », précisant « qu'ils doivent se rattacher au développement historique de Moscou et à la silhouette du futur Palais des Soviets » (p. 140). En effet, ce projet, finalement remporté par Iofan et qui devait culminer à près de 500 mètres avec à son sommet une statue de Lenin, avait été interrompu par la guerre, mais il n'était toujours pas abandonné. Le réaménagement de Moscou fut supervisé par Aleksandr Vlasov (1900-1962) qui avait réalisé le parc Gor'kij. Il fut nommé dès 1945 membre de l'influent Conseil d'État pour l'architecture, puis architecte en chef de Moscou de 1950 à 1955.

Sept gratte-ciel staliniens furent finalement réalisés. Le plus réussi est sans doute le ministère des Affaires étrangères dessiné en 1948 par Vladimir Gel'frejh (1885-1967) et Mihail Minkus (1905-1963). Ils conçurent leur projet comme une tour pyramidale qui évoque les réalisations du cabinet Holabird et Root, notamment le Cornell Hospital de New York construit en 1932. Toutefois, Gel'frejh et Minkus adoptèrent un style plus dépouillé qui dégage davantage la tour centrale. Ils donnèrent au bâtiment une tonalité slave par l'introduction sur les parapets du « créneau polonais » que l'on retrouve sur plusieurs kremlins en Russie ou sur la Halle aux draps de Cracovie. Seule dépare quelque peu la courte flèche terminale que Stalin imposa en personne, en dépit d'une lettre courageuse qu'osèrent lui envoyer les deux architectes. L'université Lomonosov est plus éclectique. Ce projet, d'abord attribué à Iofan, lui fut retiré et confié à Lev Rudnev (1885-1956). Celui-ci reprit à Iofan l'idée d'un plan en $\mathrm{H}$ et d'une tour centrale comme celle de l'université de Pittsburg construite en 1931 par Charles Klauder (1872-1938). Toutefois, Rudnev se différencia de ce modèle par l'adjonction d'autres références, pointes gothiques, colonnes rostrales vénitiennes, bassin qui rappelle le Grand Canal de Versailles. Les autres tours staliniennes sont plus conventionnelles. 
L'hôtel Ukrajna de Mordvinov et Oltarževskij et l'hôtel Leningradskaja de Leonid Poljakov (1906-1965) sont fort proches de leurs modèles américains du cabinet Schultze et Weaver, tels le Waldorf-Astoria édifié en 1931 à New-York. Dmitrij Čečulin (1901-1981) réalisa, selon un plan en W, le vaste immeuble d'habitation destiné à la Nomenklatura du quai des Chaudronniers. Promis à une carrière à succès, Mihail Posohin (1910-1989) construisit un autre gratte-ciel d'habitation place de l'Insurrection, pour lequel il reçut le prix Stalin. Par la suite, pour manifester l'homogénéité du bloc socialiste, d'autres tours furent érigées dans les pays sous influence soviétique. La plus importante d'entre elles est le Palais de la culture construit à Varsovie de 1952 à 1954 sous la supervision de Lev Rudnev. On peut y ajouter l'Académie des sciences de Riga, la Casa Scînteii de Bucarest ou l'hôtel International de Prague. En effet, au moment où le discours jdanovien condamnait toute appréciation de l'Amérique, la production architecturale soviétique parsema de fait les capitales du bloc socialiste d'une série de gratte-ciel répondant à l'adversaire désigné : « car malgré les précautions d'usage, les tours staliniennes traduisent bien un américanisme en apparence nié, mais repensé, réadapté, réinventé » (p. 179).

Après la mort de Stalin, Nikita Hruščev dénonça dès 1954 le néoacadémisme. À l'intérieur, pour répondre aux immenses besoins en logement de la population, la priorité fut réorientée vers la construction d'immeubles de quatre à cinq étages, les fameuses «hruščevki». À l'extérieur, sans doute pour en accélérer la réalisation, Hruščev n'hésita pas à faire appel à l'architecte canadien Werner Noffke (1878-1964) pour construire l'ambassade d'Ottawa². Dans les constructions de grande ampleur, les architectes soviétiques retrouvèrent davantage de liberté pour se confronter au style contemporain international, inaugurant la période de la « seconde modernité soviétique » (p. 208). Abandonnant son style néoacadémique, Mihail Posohin conçut en 1963 la tour du Comecom largement inspirée du City Hall de Toronto de Viljo Revell (1910-1964). Suite au tremblement de terre de Taškent en 1966, Evgenij Rozanov (1925-2006) reconstruisit la ville avec plusieurs grands équipements qui rappellent les réalisations de Le Corbusier à Chandigarh ou de Niemeyer à Brasilia. À Moscou, la célèbre maison de la Begovaja, conçue en 1974 par Andrej Meerson (né 1930) et Elena Podolskaja, évoque la Cité radieuse de Le Corbusier à Marseille.

Nommé en 1960 architecte en chef de Moscou, Mihail Posohin conserva ce poste jusqu'en 1982, tant par le talent incontestable dont témoignent certaines de ses réalisations que par son opportunisme. Jusqu'au milieu des années 1950, plusieurs architectes proposèrent encore des projets pour le Palais des Soviets, Vlasov selon un style constructiviste, Gel'frejh et Minkus avec une composition en longueur ornée de colonnes rappelant le Capitole de Washington. Posohin eut l'habileté d'enterrer définitivement le projet en construisant à la place le solennel Palais des Congrès du Kremlin réalisé de 1962 à 1967. Ceci nécessita la destruction de plusieurs églises du Kremlin, alors que Aleksej Ščusev (1873-1949), maître de Posohin et auteur du mausolée de Lenin, s'était toujours battu pour préserver ce patrimoine exceptionnel. De même, Posohin fit construire la nouvelle Arbat de 
1964 à 1969 en sacrifiant une grande partie du quartier historique. Toutefois, il sut faire preuve d'innovation avec son pavillon aux superstructures épurées pour l'exposition universelle de Montréal de 1967 (remonté lui aussi près du VDNH), puis avec celui de l'exposition d'Osaka de 1970, dont l'avant s'élève en forme de fusée. En 1970, également, il construisit l'ambassade à Brasilia qui s'intègre assez bien aux bâtiments de Niemeyer.

Dans sa dernière période, Posohin fut l'auteur de plusieurs réalisations de l'ère brejnévienne. Ce style se caractérise par le soin de s'éloigner des influences occidentales tout en accentuant une «monumentalisation emphatique, toujours moderne en certains points, mais réaffirmant un esprit classique désormais hypertrophié, gelé dans l'emphase... » (p. 220). En même temps que celle de Brasilia, Posohin reçut la commande de l'ambassade soviétique à Washington. Il en fit une vaste citadelle diplomatique où des immeubles administratifs séparent la ville du complexe d'habitation des diplomates et de leurs familles, cependant qu'une tour de bureaux évoque celle d'un château fort. À Moscou, il réalisa encore entre 1975 et 1985 le titanesque ministère de la Défense.

C'est à Cuba que furent édifiés les ultimes symboles de domination soviétique. À La Havane, un monument à Lenin fut achevé en 1984, œuvre du sculpteur Lev Kerbel (1917-2003) associé à l'architecte cubain Antonio Quintana Simonetti (1919-1993). En dépit de sa taille gigantesque, l'œuvre présente le seul visage de Lenin se détachant sur un bloc de pierre qui ressemble fort... au socle de la statue de Pierre le Grand à Saint-Pétersbourg. Tout aussi étrange est l'ambassade, construite par Alexandr Ročegov (1917-1988). À Moscou, il collabora à l'hôtel Leningradskaja puis réalisa en 1968 le magasin Moskovskij, près de la gare de Kazan'. Achevée en 1987, l'ambassade à Cuba est surmontée d'un édicule, mais surtout ceinturée d'énormes verrières en encorbellement qui lui valurent aussitôt son surnom de «tour de contrôle » auprès des Cubains. Fabien Bellat conclut sur ce double paradoxe. D'une part, les œuvres qui devaient être les plus démonstratrices de la puissance de l'URSS furent achevées juste avant son éclatement, de l'autre, «L'ambassade soviétique de Cuba fut le dernier grand geste monumental international de l'URSS. [...]. Ce geste architectural aura eu lieu sur le continent américain, que le régime aura à la fois tant honni et tant observé » (p. 283).

1 - Fabien Bellat, « À fleurets mouchetés : 1'architecture soviétique sous le glacis brejnévien », Cahiers du monde russe, 54 (1-2), 2013, p. 213-237.

2 - Les Soviétiques imposèrent toutefois des façades monumentales et déjouèrent la pose de micros grâce à de faux plans intérieurs.

\section{Vincent Hecquet}

Eurostat G1 